\title{
EL TRANSPORTE COLECTIVO EN LA CIUDAD DE ALICANTE Y SU ENTORNO: EVOLUCIÓN Y PROPUESTA DE FUTURO
}

\author{
Jesús Rafael De Vera Ferre
}

\begin{abstract}
RESUMEN
Los sistemas de transporte colectivo en la ciudad de Alicante y su entorno han tenido dos etapas históricas en su evolución. La primera, arrancando a finales del siglo XIX, se desarrolla principalmente en los dos primeros tercios del Novecientos, teniendo como protagonista principal a la red tranviaria, la cual, no solamente va a servir a la demanda existente, sino también va a dirigir el crecimiento y expansión de la ciudad en la periferia. La segunda, más reciente, se produce en paralelo a la eclosión del automóvil privado, y se basa en el autobús, que sufre, en la actualidad, graves problemas de inadaptabilidad.

También se realiza en el artículo una propuesta de cambio radical de los sistemas de transporte colectivo en el área metropolitana alicantina.
\end{abstract}

\begin{abstract}
Public transport in the Alicante area has evolved in two stages. The tramway network played the maine role from the late $19(\mathrm{tm})$ century up to the nineteen sixties. The trams met the existing demand for transport and guided the city's growth and expansion. In recent years, the second stage has followed in parallel the rapid increase in private cars along with the bus system. Buses are more and more unsuitable nowadays to the area needs.

This article proposes a radical change of approach to public transport systems in the Alicante metropolitan area.
\end{abstract}

\section{La supresión de los tranvías, el final de una etapa en el transporte colectivo}

Los años del desarrollismo supusieron el desmantelamiento de las redes tranviarias de las ciudades españolas, como consecuencia del auge experimentado en la industria automovilística, a la par que los precios de los combustibles presentaban competitividad y el aparato estatal y municipal se desentendía parcialmente del problema del transporte colec- 
tivo para hacer un creciente hincapié en la construcción y modernización de las infraestructuras viarias destinadas, fundamentalmente, al tráfico privado.

La ciudad de Alicante no escapó a la situación general del país. De esta forma, las líneas de tranvías fueron desapareciendo paulatinamente, al tiempo que la inversión necesaria para su modernización se desviaba hacia otros fines, considerados como más prioritarios por el municipio, abocando la explotación en una obsolescencia creciente que le restaba eficacia y que la incardinaba hacia un final anunciado al acumularse de esta forma déficits cuantiosos ${ }^{1}$.

Este hecho supuso la sustitución de los viejos tranvías por autobuses, lo que encareció el precio del billete, a la vez que se retrocedía en la eficacia de la red, ya que mientras la tranviaria tenía un marcado carácter metropolitano, al unir la ciudad capital con los municipios de San Vicente, por un lado, y de San Juan de Alicante y Muchamiel, por otro, la nueva estructura aparecía como claramente fragmentaria y descoordenada, al establecer dos sistemas paralelos y, en gran medida, coincidentes, lo que suponía duplicación de servicios: una empresa se hacía cargo de las líneas del casco urbano, mientras que otra distinta llevaba a cabo la explotación de las metropolitanas, que, a diferencia de la situación anterior, ya no estructuraban la red arterial colectiva, a la par que no penetraban en el núcleo central, fijando sus terminales a las puertas del mismo. La plaza de España, en la zona Norte, quedó constituida como el final de trayecto para la conexión con San Vicente, mientras que la Puerta del Mar, a mediodía, lo hacía para la de Muchamiel.

\section{Los sistemas de transporte colectivo basados en la hegemonía de la red tranviaria}

El cierre del servicio tranviario suponía la finalización de un largo y complejo proceso de dotación ala ciudad y su alfoz de una red de transporte colectivo que satisfaciera las crecientes necesidades de movilidad en una época en que la misma estaba ligada inexcusablemente a un servicio de tipo comunitario.

El primer intento de creación de un tranvía de vapor se produce hacia 1881. El ayuntamiento de la capital declara de utilidad pública el que habría de enlazar a la misma con Elche y Crevillente ${ }^{2}$, aunque el proyecto no cristalizaría hasta $1904^{3}$.

La conexión con la huerta, a través de la Santa Faz, San Juan y Muchamiel, entra en servicio, con coches de tipo Ripper, mucho antes, en $1889^{4}$, cubriendo así una fuerte demanda de transporte que, hasta ese año, solamente había podido ser satisfecha con carros.

El último decenio del Ochocientos supone la puesta en ejecución del proyecto de tranvía de mulas a Benalúa ${ }^{5}$, barrio situado a occidente de la ciudad consolidada y cuya accesibilidad era difícil debido a su separación del casco urbano por el barranco de San Blas, normalmente

1 En 1955, la recaudación de la red tranviaria alcanzó casi diez millones de pesetas, sin embargo, en 1967, a pesar del aumento de la inflación, solamente se eleva a 13'8 millones. En cambio, los autobuses pasan de 222.000 pesetas a 50'8 millones. Para mayor información, vid. DE VERA, J.R., Estudio Geográfico del tráfico urbano de Alicante en los primeros años setenta, Alicante, Caja de Ahorros Provincial, 1985, pp. 144 y 145.

2 AMA, Acta de 30 de septiembre de 1881.

3 El servicio se autoriza por Real Orden de 8 de noviembre de 1904, abriendose al público en 1905.

4 RAMOS, V., Historia de la provincia de Alicante y de su capital, Alicante, Diputación Provincial, 1971, T. II, p. 110 .

5 GIL, F., Crónicas alicantinas, Alicante, CAAM, 1977, pp. 162 y ss. Se inauguró el 12 de julio de 1893. 


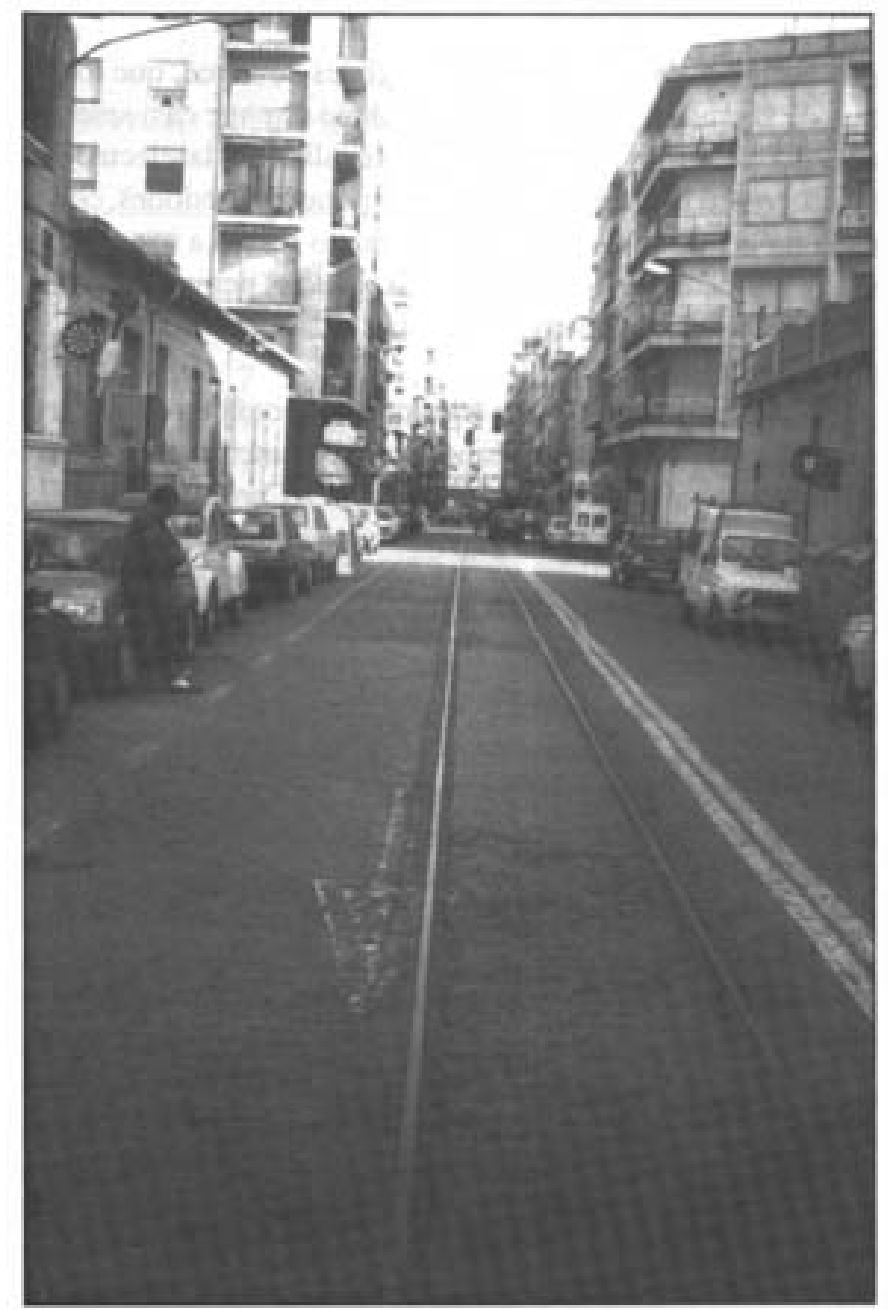

Fotografía 1

La calle Pintor Gisbert creció en gran medida gracias al trazado del tranvía de San Blas. Veinte años después de desaparecido el servicio, los railes siguen estando presentes en su calzada.

arreico, pero que recobraba su papel de conductor de la escorrentía con motivo de la existencia de lluvias de fuerte intensidad horaria, que inutilizaban varias horas los difíciles enlaces.

A finales de 1901, se comienza a construir el tranvía con tracción animal de la Huerta, puesto en servicio en 1902, pasando a ser de vapor un año más tarde, mientras que en 1906 se inaugura el de San Vicente ${ }^{6}$.

6 RAMOS, V., Opus cit., t. II, pp. 178,179 y 214. 
Una vez puestas las bases de los grandes ejes axiales de la red, que iban a estructurar el transporte colectivo de la ciudad y su entorno inmediato durante casi sesenta años, se inicia un proceso de modernización de la misma, que cristaliza en la electrificación. El ayuntamiento de la ciudad aprueba la unificación de las distintas concesiones, con el fin de facilitar la explotación mediante la nueva tecnología, celebrando sesión a instancia de la Compañía General de Tranvías y Ferrocarriles Vecinales de España ${ }^{7}$, al tiempo que la Real Orden de 23 de noviembre de 1907 autorizaba la misma. Sin embargo, su puesta en práctica se hizo esperar hasta la década de los veinte ${ }^{8}$.

El constante aumento de la demanda de transporte suponía un crecimiento del servicio colectivo. La primera línea de autobuses urbanos entra en funcionamiento en $1924^{9}$, mientras que en los años siguientes se amplía la malla tranviaria a Carolinas, San Blas y La Florida Alta. También por estas fechas se ponen en servicio los autobuses con destino a Villafranqueza y Campello, siendo este último complementario a la línea férrea, de vía estrecha, de La Marina.

La red de transporte colectivo se mantiene estabilizada hasta la postguerra, cuando de la arteria tranviaria que unía Alicante con su huerta se deriva una afluente a la misma que, de forma capilar, drena más eficientemente la zona del Plá del Bon Repós, a la vez que se enlazaba el Hospital Provincial con el centro urbano ${ }^{10}$.

Por la misma época se establece una conexión, mediante autobús, con el barrio de San Gabriel, muy deficientemente comunicado hasta entonces, debido a lo alejado del núcleo principal y por las barreras que establecía para su acceso la fábrica CROS y el barranco de las Ovejas ${ }^{11}$. Un poco más adelante, en los años cincuenta, se pone en servicio, por el mismo sistema, la que enlaza el Mercado Central con Ciudad de Asís. En los primeros años sesenta, el espectacular crecimiento de la ciudad provoca la aparición de varias líneas de autobuses que enlazarán barrios en proceso de adensamiento, como Divina Pastora o la Albufereta, o de nuevo cuño, como Mil Viviendas y Virgen del Remedio, área hacia la que se prolongará también el tranvía de Carolinas ${ }^{12}$. Precisamente este tranvía será el único caso de rompimiento de la red radial, al fusionarse en aquellos años con el de Benalúa, uniendo los dos extremos del casco consolidado lo que le daba una alta rentabilidad.

En los últimos años de funcionamiento de la red tranviaria, las líneas de autobuses conviven con las eléctricas, superando con creces el kilometraje de las segundas ${ }^{13}$.

En el cuadro siguiente, quedan reflejados algunos datos de interés de la explotación tranviaria en los últimos años de servicio de la misma.

En el año 1969, tras cuatro años en que el servicio había quedado muy disminuido en lo

7 AMA, Acta del 5 de julio de 1907.

8 En 1923, se inaugura la electrificación del tranvía de la Huerta; un año más tarde, el de San Vicente y los urbanos del área occidental de la ciudad.

9 AMA, Acta del 7 de mayo de 1924.

10 En el bienio 1956-57, se alcanza el máximo número de líneas de tranvías, cuya numeración y destino era el siguiente: 1, Benalúa; 2, Carolinas; 3, Ciudad Jardín y San Vicente; 4, Vistahermosa, Santa Faz, San Juan y Muchamiel; 5, San Blas; 6, Plá Hospital; 7, Florida Alta y Baja; y 8, Benalúa, por Alfonso el Sabio.

11 Dicho barrio había empezado a construirse en 1930.

12 El tranvía 2 enlazará, a partir de mediados de la década, Virgen del Remedio, Francisco Franco y Carolinas Altas y Bajas con el Mercado Central y Benalúa.

13 En 1967, frente a 19 kilómetros de red tranviaria existen 118 de autobuses. 
Cuadro I

EL SERVICIO TRANVIARIO EN ALICANTE DE 1955 A 1967

\begin{tabular}{cccc}
\hline Años & $N^{2}$ líneas & Km. líneas & $N^{2}$ viajeros (en miles) \\
\hline 1955 & 7 & 32 & 16.796 \\
1956 & 8 & 32 & 18.140 \\
1957 & 8 & 33 & 18.654 \\
1958 & 7 & 30 & 19.635 \\
1959 & 7 & 30 & 21.420 \\
1960 & 7 & 30 & 21.950 \\
1961 & 7 & 30 & 20.839 \\
1962 & 7 & 30 & 22.248 \\
1963 & 7 & 25 & 22.583 \\
1964 & 7 & 25 & 19.599 \\
1965 & 6 & 25 & 18.949 \\
1966 & 3 & 19 & 15.617 \\
1967 & 3 & 19 & 13.416 \\
\hline
\end{tabular}

Fuente: I.N.E., Reseña Estadística de la provincia de Alicante, Madrid, 1969.

concerniente al número de líneas, el tranvía dejó de circular por las calles alicantinas, siendo sustituido por autobuses. El último día de funcionamiento fue el 25 de noviembre.

\section{El impacto de la red tranviaria en la ordenación del territorio alicantino}

Una cuestión no abordada todavía en los estudios geográficos de la ciudad de Alicante es el impacto de las líneas de tranvías en el proceso de crecimiento y ordenación de la malla urbana. Si es evidente que las carreteras de acceso a la ciudad han tenido un protagonismo en la estructuración de buena parte de la periferia, no es menos cierto que el trazado de la red tranviaria, signó de forma clara el devenir de algunos espacios, alejados en aquel momento del casco consolidado, y que, por carencias tecnológicas de los sistemas de transporte, se mantenían al margen del mercado inmobiliario. El tendido de la vía supuso el rompimiento de su falta de accesibilidad y la revalorización del precio del suelo que, a pesar de ello, en la mayoría de los casos, se mantenía a un nivel inferior al de los barrios centrales.

En las líneas que enlazaban la zona occidental de la periferia con el centro urbano hay ejemplos palmarios del impacto del trazado tranviario sobre el espacio geográfico por el que discurre. Así, la calle Asturias, en la Florida, eje de penetración de la red desde Benalúa, se convierte en el principal motor de la expansión del barrio, a través de modestas casas de planta baja, quedando incluso hoy configurada como una de las principales arterias de la zona a la que afluyen un gran número de calles secundarias.

En el Ensanche, el trazado de la línea a Benalúa supuso que la calle Ramales -hoy, de Reyes Católicos- se poblara mucho más rápidamente que las colindantes -a excepción de Maisonnave, que era la salida hacia Madrid-, lo que, sin duda, se debe a que por aquella penetraba el tranvía en el casco urbano consolidado, enlazando con el arrabal de San Francisco, en su discurrir hacia el centro. 


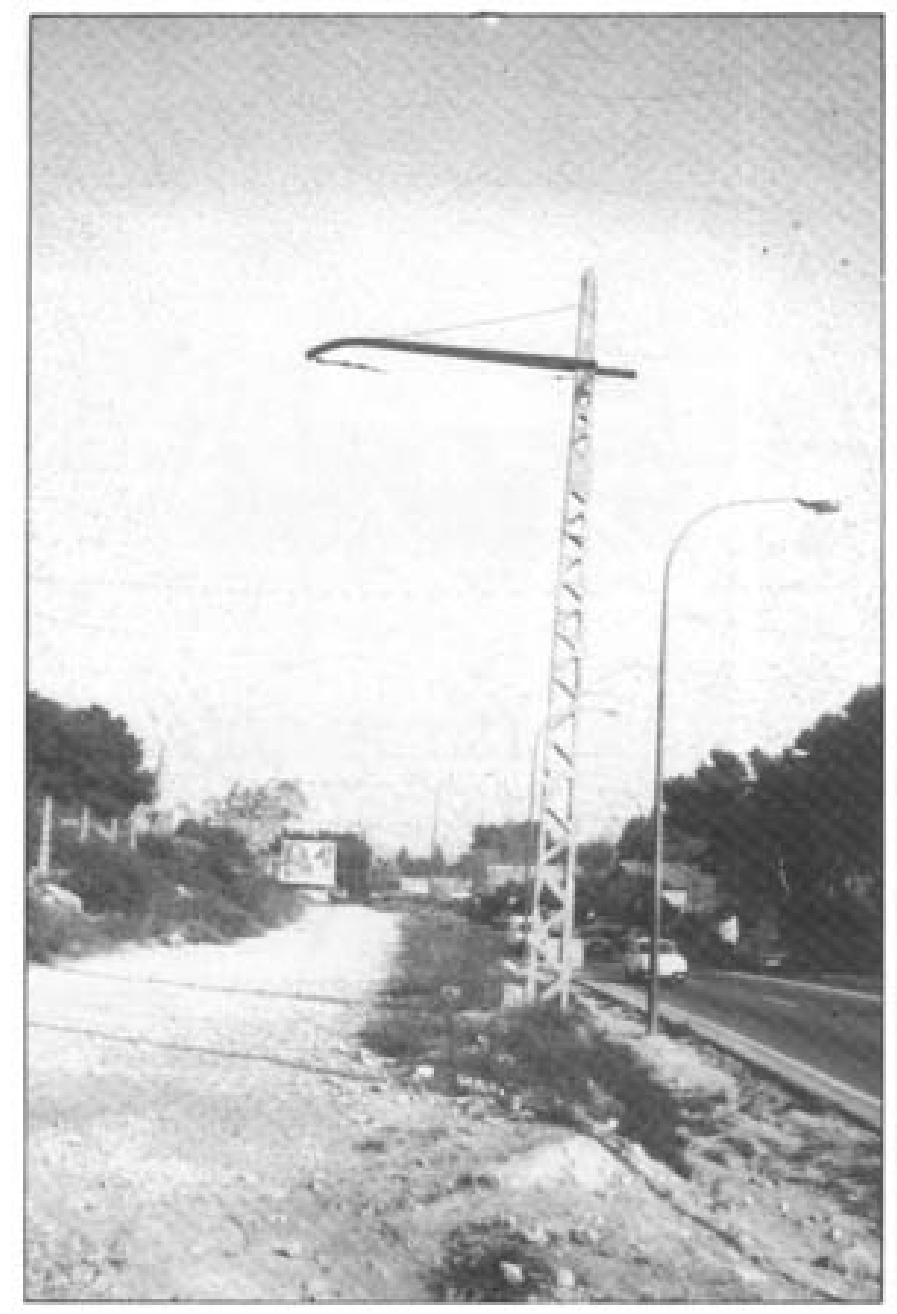

Fotografía 2

Junto a la carretera de Valencia, en Vistahermosa, cerca del colegio de las Teresianas, es visible todavia hoy la plataforma sobre la que discurría el tranvía número 4 o de la Huerta, asi como los postes que sustentaban el tendido eléctrico.

En la línea que unía Alicante con San Juan y Muchamiel existen también varios ejemplos de expansión de la ciudad por mejora de la accesibilidad. El establecimiento del servicio supuso la inmediata reordenación del barrio del Plá, que quedaba drenado por la misma, hecho que se materializa, mediante un plan de alineaciones, en 1912. Igualmente, la actual calle de Padre Esplá y el Fondó Roenes, espacios sobre los que se tienden las vías, se convierten, primero, en el eje sobre el que se asientan chalets y casas de campo y, más tarde, en los años sesenta y setenta, en el principal viario de la zona que jerarquiza, de forma clara, gran parte de la misma. 
También el tranvía de la huerta hizo posible la instalación a su vera del barrio obrero, comenzado a construir en 1919, en terrenos muy alejados de la ciudad y difícilmente accesibles. En 1921, en Vistahermosa, núcleo de chalets que creció al calor de la misma línea, se empieza a formar y consolidar la actual calle del Camarada Llopis que arranca, en perpendicular, del lugar dónde se situaba un importante cruce de vías.

El tranvía de San Vicente permitió no solamente el crecimiento de villas en el Altozano, y el nacimiento de asentamientos obreros en las zonas que drenaba, sino también la construcción de la Ciudad Jardín, creada en 1920, apoyada sobre una importante parada del mismo.

En torno a las lineas del área de Carolinas se fueron consolidando importantes ejes urbanos. El de mayor longitud, conformado por las calles de Sevilla y Espartero, coincide con el trazado tranviario. Lo mismo cabe afirmar de la calle San Carlos, por dónde bajaban los vehículos que enlazaban con el Hospital, y la del Garbinet por la que discurría el trazado hacia la Bola de Oro.

Por su parte, en San Blas lo mismo cabe indicar del importante eje jerarquizador del barrio, la calle Pintor Gisbert, por el que transitaba el tranvía de la zona.

Así, pues, la red tranviaria tuvo un importante papel en la creación y consolidación de buena parte de la periferia de la ciudad antes de que hiciera eclosión el motor de explosión que iba a transformar, de forma radical, los desplazamientos en la urbe tanto en un plano cualitativo como cuantitativo.

Gracias al tranvía fue posible ampliar la accesibilidad de los terrenos próximos a la ciudad, incluyendolos en el mercado inmobiliario, generando plusvalías crecientes, al tiempo que, lentamente, se iban produciendo múltiples interrelaciones entre la ciudad capital y los pueblos próximos, diversificando de esta forma los primeros contactos, basados fundamentalmente tanto en las necesidades de aprovisionamiento recíproco, como en las de movilidad del mercado de trabajo, cuyo ejemplo palmario lo constituye el ir y venir de las cigarreras, desde las zonas de huerta, hacia el centro fabril de la antigua Casa de Misericordia de la calle Sevilla.

El desmantelamiento progresivo de las líneas tranviarias y el cierre definitivo del servicio en 1969 se produce en aras de una pretendida modernidad que no encierra más que la necesidad de dar prioridad casi absoluta al automóvil privado, como modelo de transporte que consagra el viejo ideal del capitalismo y de la burguesía de contar con abundante mano de obra, gracias a su creciente movilidad, empuje a la industria automovilística y empresas subsidiarias, y la posibilidad de consumir gran cantidad de espacio, lo que supone el lanzamiento definitivo del mercado inmobiliario y especulativo, al tiempo que se amplía la selectivización de la ciudad según los tipos de renta de sus habitantes.

Entre los argumentos que se barajaron en la prensa de la época para justificar la desaparición del servicio se citaban la obsolescencia del mismo, a pesar de que en los últimos años se habían adquirido varias unidades nuevas del material rodante, los déficits acumulados, que se producían por la creciente motorización y por la frecuente existencia de líneas de autobuses paralelas, los defectos en la explotación, como el habitual corte de energía cuando se producían lluvias y, sobre todo, que molestaba a la circulación del automóvil privado.

Una vez suprimido el servicio, se aprovecharon algunos tramos del mismo para ampliar avenidas o abrir otras nuevas. Así, por ejemplo, los ensanchamientos de las calzadas de la avenida de Aguilera, carretera de San Vicente y Padre Esplá se realizaron por este 


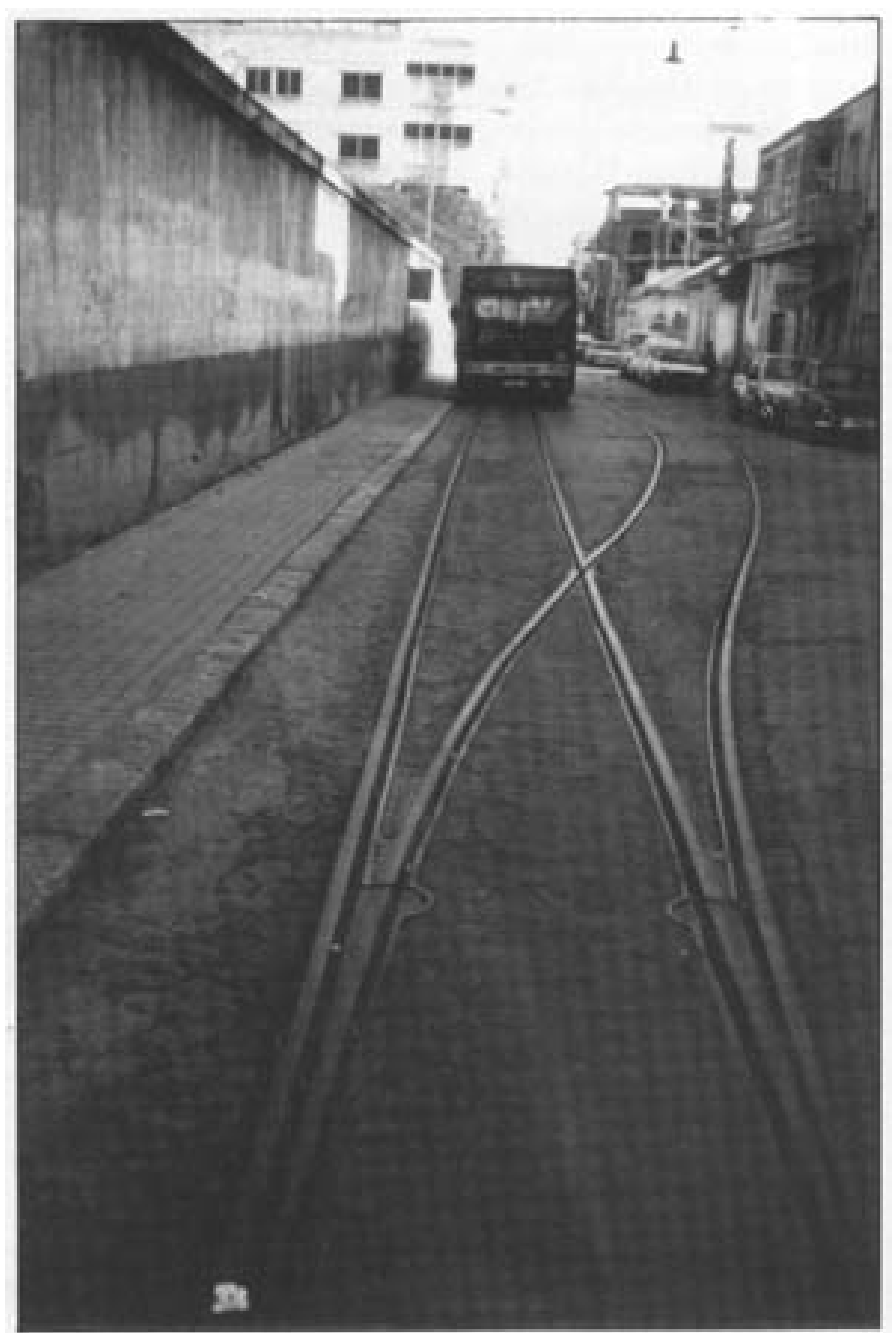

Fotografía 3

En gran medida, las actuales líneas de autobuses urbanos reproducen el antiguo trayecto de los tranvías. En la fotografía, el importante cruce de tranvías de la calle Asturias, en la Florida, en el itinerario número siete, sigue siendo la terminal del autobús $G$.

procedimiento. El actual trazado de la prolongación de esta última arteria y de Alonso Cano han nacido gracias al levantamiento de las vías tranviarias.

\section{EI monopolio del autobús en los transportes colectivos}

En el año 1969, la empresa MASATUSA (Marco y Sánchez, Transportes Urbanos S.A.), obtiene una concesión administrativa de los autobuses urbanos por parte del Ayuntamiento de la capital, mediante gestión indirecta, que estará vigente hasta el veintisiete de junio del 
2019, fecha en la que revertirá al municipio. La empresa lleva a cabo, en un primer momento, una explotación del servicio con líneas de tipo radial, cuyo punto de correspondencia es el Mercado Central. En el año 1973 se produjo un conflicto de competencias entre el Ayuntamiento y las líneas de concesión estatal, lo que movió a la Corporación a promover la realización de un trabajo técnico con el fin de delimitar el casco urbano a efectos de transporte ${ }^{14}$.

En mayo de 1976 se crean los primeros carriles de uso exclusivo para los autobuses y taxis que en la actualidad presentan una longitud de 3'1 kilómetros, con una intensidad aproximada de uso de cuarenta vehículos por hora. En el bienio 1980-81, se produce una modificación importante en las líneas gestionadas por MASATUSA, al pasar del anterior sistema radial a otro de tipo diametral que permite, con el mismo precio del billete, y sin hacer trasbordos, el enlazar los barrios extremos de la ciudad. El punto de correspondencia de todas ellas sigue siendo el Mercado Central ${ }^{15}$. La reforma supone pasar de dieciocho rutas a once, en un primer momento, que luego se ampliarían en dos más, con una longitud aproximada de 150 kilómetros.

De las trece líneas, dos enlazan la zona occidental de la ciudad con la oriental; el mismo número comunica a los barrios del Norte con el Oeste y las demás, partiendo del centro se dirigen hacia el Norte, seis; al Este, una, y al Oeste, dos.

A pesar de las mejoras en el servicio, que no solamente cristalizan en la renovación de las líneas, sino también en una mejor frecuencia y una flota que es de las mejores de España en calidad y juventud, el servicio de autobuses cada vez transporta menos pasajeros. Si en 1971, utilizaron la red casi 37' 5 millones de personas, seis años más tarde se había reducido a 26 ' 7 y en 1980, a 24'7. En 1987, la cifra de viajeros estuvo en torno a los veinte millones y para 1988, se calcula, según fuentes de la propia empresa, una disminución global del 1'5\% de usuarios.

\section{La dimensión metropolitana del transporte alicantino}

Un tema pendiente en el mundo del transporte colectivo de viajeros en la ciudad de Alicante es el de la coordinación de los servicios dentro del propio municipio y con los del área metropolitana.

En la actualidad, junto a MASATUSA, tienen actividad en el sector la empresa ESCOLANO en los enlaces con Villafranqueza y Tángel y con el aeropuerto, éste último en término municipal de Elche; AUTOBUSES DE LA PLAYA DE SAN JUAN que conecta con el sector costero oriental y con Campello; y LA ALCOYANA que tiene la concesión de las líneas que cubría la antigua red tranviaria a San Juan y Muchamiel, por un lado, y a San Vicente, por otro, que comunica a la Universidad con la ciudad capital.

Sin embargo, existe un proceso en marcha de concentración empresarial. En 1974, MASATUSA llega a un acuerdo con AUTOBUSES DE LA PLAYA DE SAN JUAN y en 1988, se alcanza una situación similar con LA ALCOYANA.

14 Es el denominado Plan de coordinación de transportes, realizado por INECO (Ingeniería y Economía de Transporte), Alicante, inédito, 1973.

15 Esta reorganización fue el fruto de un trabajo realizado entre octubre de 1979 y abril de 1980. OLIVAS, D. y ASILA, J.M., Estudio para la reorganización del transporte urbano de Alicante, Alicante, inédito, 1980. 
Aunque está en estudio desde hace años la creación de un Consorcio de Transportes para el área metropolitana, que promovido por la Consellería de Obras Públicas de la Generalitat Valenciana, tiene como fin el llegar a una coordinación similar a la que se ha conseguido en la ciudad de Valencia, el proceso avanza muy lentamente, no habiendose plasmado en un documento definitivo en el momento de escribir este artículo.

De esta forma, la explotación de los autobuses se presenta como inadecuada a la realidad socioeconómica y demográfica que ha de satisfacer, por inexistencia de servicios en unos casos, duplicidad en otros, ineficacia en el enlace de los distintos municipios entre sí y con el centro de servicios de la ciudad capital y falta de homogenización en las tarifas y en la gestión, por no hablar de las desigualdades en la calidad prestada ${ }^{16}$.

Hay una serie de hechos objetivos que avalan la urgente necesidad de coordinar y mejorar el transporte colectivo en el área metropolitana. Por un lado, el crecimiento demográfico experimentado. Si en 1970, la misma contaba con 216.225 habitantes, en 1975, sumaba 255.369, para pasar, en el último censo, a 295.452, que han ascendido, en 1986, a 312.413 ${ }^{17}$.

Por otro lado, el grado de complejidad que está alcanzando el territorio, al calor del crecimiento turístico de las playas, que aumenta el número de habitantes en los meses estivales, la saturación del municipio central y el costo creciente de su suelo, la expulsión de las actividades industriales hacia San Vicente, la creciente urbanización de los antiguos terrenos de huerta, la instalación de importantes centros universitarios, sanitarios y de distribución comercial en los términos de San Vicente y San Juan, y la creciente especialización de Alicante, han variado de tal forma el tipo, dirección y cantidad de los desplazamientos de los habitantes de la zona que es preciso adecuar la oferta a la demanda.

\section{Una propuesta de futuro para el transporte metropolitano de Alicante}

A pesar de las grandes obras de infraestructura en construcción o en proyecto, tales como la autovía de circunvalación, la Gran Vía, la Vía Parque y el desdoblamiento de la AV-2.203, el área metropolitana alicantina ha de buscar, a largo plazo, nuevas soluciones al transporte, si no quiere verse colapsada, ya que el grado de motorización es muy alto.

A 31 de diciembre de 1987, había matriculados en la provincia de Alicante 640.000 vehículos, de los que se calculan que circulan medio millón. En ese mismo año se incorporaron 46.655 nuevas unidades, lo que da un promedio de cerca de 4.000 matrículas al mes ${ }^{18}$.

Solamente en la ciudad de Alicante, existen a fines de 1988, más de 112.000 vehículos, con un ritmo de crecimiento anual superior a los 7.000. Casi un 66 por ciento de los hogares posee automóvil, en los que se realizan el 32 ' $1 \%$ de los deplazamientos ${ }^{19}$.

Las cifras son lo suficientemente elocuentes para aconsejar variar el modelo de transporte

16 Vid. DE VERA, J. R., "El transporte colectivo en el área metropolitana alicantina”, Información, 9 de junio de 1987, p. 2.

17 El área metropolitana está conformada por los municipios de Alicante, San Vicente, San Juan, Muchamiel y Campello.

18 Datos facilitados por la Jefatura Provincial de Tráfico.

19 SÁNCHEZ-CASAS, C., et al., "Análisis específicos: tráfico y transporte”, Información urbanistica y avance del planeamiento para la revisión del Plan General Municipal de Ordenación Urbana de Alicante. Alicante, inédito, s.a., t. 2. 


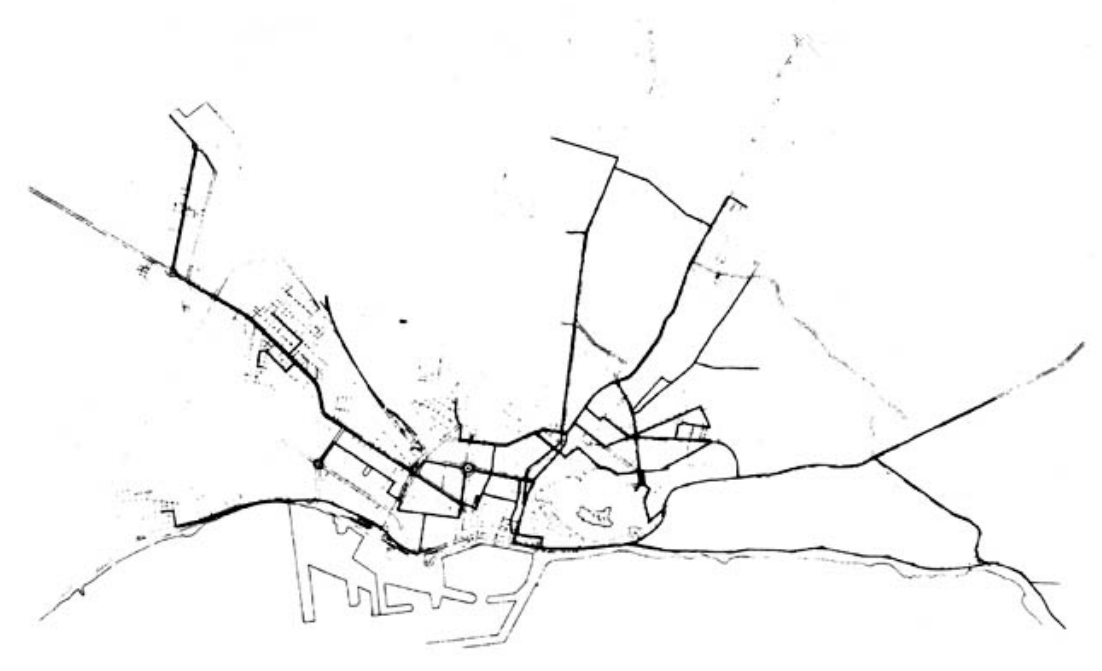

Gráfico 1. Esquema básico de la red de autobuses de Alicante

colectivo. Por ello, quizá sería conveniente el plantearse unos estudios de viabilidad para la implantación de una red tranviaria, de cuño moderno, con trazado separado de la red general, que uniera los distintos municipios y de la cual arrancarían una serie de líneas de autobuses que se distribuyeran capilarmente por todo el tejido urbano.

El trazado definitivo de la red tranviaria no tendría que agotarse en planteamientos de simple oferta y demanda, tan al uso, sino que debería enfocarse también como un medio de ordenar el territorio de forma equilibrada, ya que la misma, por su alto poder estructurarte, variaría los precios y usos del suelo, al marcar los itinerarios básicos y las zonas de correspondencia y cambiaría ostensiblemente la accesibilidad de amplias zonas. 